Math. Model. Nat. Phenom.

Vol. 7, No. 1, 2012, pp. 279-305

DOI: $10.1051 / \mathrm{mmnp} / 20127113$

\title{
Mathematical Modelling of Cancer Stem Cells Population Behavior
}

\author{
E. Beretta ${ }^{1}$, V. Capasso ${ }^{1 *}$ and N. Morozova ${ }^{2}$ \\ ${ }^{1}$ CIMAB (InterUniversity Centre for Mathematics Applied to Biology, \\ Medicine and Environment) \\ Dipartimento di Matematica, Universitá degli Studi di Milano, 20133 Milano, Italy \\ ${ }^{2}$ CNRS FRE 3377, Laboratoire Epigenetique et Cancer, CEA Saclay \\ 91191 Gif-sur-Yvette, France
}

\begin{abstract}
Recent discovery of cancer stem cells in tumorigenic tissues has raised many questions about their nature, origin, function and their behavior in cell culture. Most of current experiments reporting a dynamics of cancer stem cell populations in culture show the eventual stability of the percentages of these cell populations in the whole population of cancer cells, independently of the starting conditions. In this paper we propose a mathematical model of cancer stem cell population behavior, based on specific features of cancer stem cell divisions and including, as a mathematical formalization of cell-cell communications, an underlying field concept. We compare the qualitative behavior of mathematical models of stem cells evolution, without and with an underlying signal. In absence of an underlying field, we propose a mathematical model described by a system of ordinary differential equations, while in presence of an underlying field it is described by a system of delay differential equations, by admitting a delayed signal originated by existing cells. Under realistic assumptions on the parameters, in both cases (ODE without underlying field, and DDE with underlying field) we show in particular the stability of percentages, provided that the delay is sufficiently small. Further, for the DDE case (in presence of an underlying field) we show the possible existence of, either damped or standing, oscillations in the cell populations, in agreement with some existing mathematical literature. The outcomes of the analysis may offer to experimentalists a tool for addressing the issue regarding the possible non-stem to stem cells transition, by determining conditions under which the stability of cancer stem cells population can be obtained only in the case in which such transition can occur. Further, the provided description of the variable corresponding to an underlying field may stimulate further experiments for elucidating the nature
\end{abstract}

\footnotetext{
*Corresponding author. E-mail: vincenzo.capasso@unimi.it
} 
of "instructive" signals for cell divisions, underlying a proper pattern of the biological system.

Key words: cancer stem cells, delay differential equations, qualitative behavior, stability, oscillations

AMS subject classification: 92C37, 34C99, 34K06

\section{Introduction}

According to their biological definition, stem cells are cells with two specific features - the ability to differentiate into all range of specialized cell types and the ability to renew themselves. There are two distinct types of mammalian stem cells: embryonic stem cells that are totipotent, e.g. having the possibility to differentiate into all specialized tissues of the developing organism, and the adult stem cells, which are multipotent, meaning the possibility to substitute specialized cells of the corresponding tissues, thus maintaining the normal turnover of it. The adult stem cells are found in adult tissues in specific stem cells niches, and their number within the corresponding tissue is generally very small (Watt \& Hogan (2000), Weinberg (2007)).

The current concept about stem cells of adult organisms is that, unlike differentiated cells, they undergo asymmetric cell division producing only one stem cell (for renewing) and one differentiated cell with the features of the corresponding tissue. Generally speaking, the differentiated cell from first division is named progenitor cell, and also has some limited self-renewal potential; progenitor cells may go through several rounds of cell division before being terminally differentiated.

Recent discovery of cancer stem cells in tumorigenic tissues has raised plenty of debates about their nature, origin, possible connection with "normal stem cells" and even about the possibility to use this term (cancer stem cells), if its biological nature is not completely elucidated yet, and there is a possibility that they may have nothing in common with "normal stem cells".

The current conventional statement is that cancer stem cells (CSCs) constitute a subpopulation of cells within tumors that could actively drive tumor growth and recurrence (Reya et al. (2001); Bao et al. (2006); Zhang et al. (2008); O'Brien et al. (2007); Ricci-Vitiani et al. (2007); Li et al.(2007); Dean et al. (2005)). Initially, cancer stem cells can be determined only operationally by their ability to seed new tumors, and, for this reason, they have also been termed "tumor-initiating cells" (Bao et al. (2006)). However, for some cancer cell lines the specific cancer stem cell markers were reported, which allow biochemical determination of this population and its further analysis. This has led to recent identification of CSCs in hematopoietic, breast, colon, ovary, brain, pancreas, and prostate cancers (Ginestier \& Wicha (2007); O'Brien et al. (2007); Zhang et al. (2008); Li et al. (2007); Maitland \& Collins (2008); Gimble et al. (2007); Gupta et al. (2009)); Bonnet \& Dick (1997)). By summarizing results of recent studies, we may list the following important properties of cancer stem cells:

- CSCs present a small subpopulation of cells within tumors capable to cause tumor growth (Ginestier \& Wicha (2007); O’Brien et al. (2007); Zhang et al. (2008); Clarke et al. (2006)); 


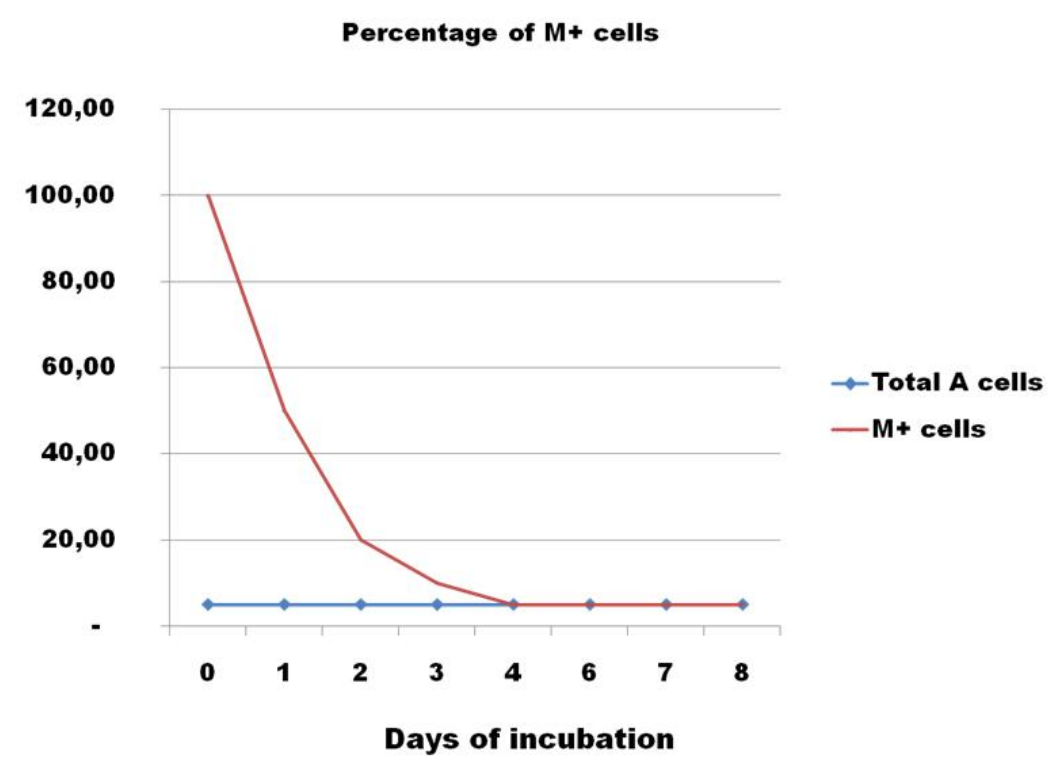

Figure 1: Schematic representation of the cancer stem cell subpopulation $(\mathrm{M}+)$ dynamics in the whole cancer stem cell population (blue), and in purified $\mathrm{M}+$ subpopulation (red), according to numerous experimental data in cell culture (e.g. C. Ginestier, V. Maguer-Satta, A. Harel-Bellan, personal communications). Cancer stem cells were marked using specific cancer stem cell markers (M).

- CSCs have asymmetric cell divisions like normal stem cells providing self-renewing ( Mani et al. (2008); Ginestier \& Wicha (2007); Clarke et al. (2006));

- CSCs can be isolated with cell-surface marker profiles (Zhang et al. (2008); Lang et al. (2009); Dean et al. (2005); O’Brien et al. (2007); Maitland \& Collins (2008)).

The very important observation made in works on several cancer cell lines is that the percentage of cancer stem cell population is maintained at the same level during several years of passages (Figure 1, blue curve). Another important observation is that when a cancer stem cell population is isolated, purified and maintained in culture, the percentage of stem cells rapidly decreased and finally stabilized at the initial level, characteristic for this given cell culture (Figure 1, red curve).

These experimental data raise two questions: do there exist "instructive signals" for maintaining this population behavior? If yes, what is the nature of this "instructive signals"? In our work we would like to address the first question and suggest some ways for its solution using mathematical modelling of cancer stem cells evolution. The suggested model is based on the main biological features of cancer stem cells behavior, supplemented by the description of cell-cell communication 
using the concept of an underlying field. We hope that next the results of mathematical modelling can enable experimentalists to design the proper search of the molecular mechanisms adequately solving the second question.

Various mathematical models have been recently proposed for describing the dynamics of stem cell populations (see e.g. Roeder et al. (2009), Zhdanov (2007), D'Onofrio \& Tomlison (2007), and references therein), and some of them (see Michor et al. (2004)), also consider cell-cell communication. However, these works do not address the question about cancer stem cell population stability, and suggest different approaches for the description of cell population dynamics.

\subsection{The main assumptions}

Our main goal is to create a mathematical model describing the phenomena of cancer stem cell population stabilization. Our first statement is that the evolution of the structure of a whole cell population (in our case, the population structure means just a percentage of stem cells in it) depends on the pattern of cell divisions, on the speed of cell divisions in both stem and non-stem cell populations, and on the rates of cell death. Our main assumption is the existence of an underlying field, carrying the information about the population structure and influencing the pattern and speed of cell divisions in the given conditions. Thus, there are two biological points which we considered as the main ones for building the mathematical model of cells population behavior:

1. The type of cell divisions.

2. The speed of cell divisions.

It is very important to mention that, with all differences in normal (non cancer) and cancer stem cells nature, and independently from the experimental methods of their investigation (mostly in vitro (in cell culture) for cancer stem cell and mostly in vivo for normal stem cells), the two main statements, which we consider as a basic ones for our modelling, are the same in both cases:

- as a rule, stem cells undergo asymmetric divisions with relatively slow rates, while non-stem cells undergo symmetric divisions with relatively quick rates.

- the main feature of stem cells population behavior is the tendency of maintaining the proper (very small) percentage of stem cells in the whole tissue (culture).

Thus it is clear that, though our main objective is the modeling of cancer cell population behavior, the main general idea of the model - the mathematical description of the main features of the "instructive signals" from the whole system (tissue or culture) to its cells for maintaining the proper population pattern - can be applied also to normal (non cancer) stem cells population behavior. In this cases "non-stem cells" in our model should be understood as differentiated cells of normal tissues. It is important to note, that in cancer tissues/cultures non-stem cells can not be named "differentiated", as carcinogenesis strongly influences the proper cell differentiation.

Considering the types of cell divisions, we can point out that, among several possible scenarios, the asymmetric cell divisions, providing self-renewing, is considered to be the main one. However, 


\section{Usually suggested}

1

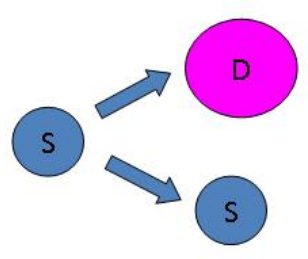

Other possible modes

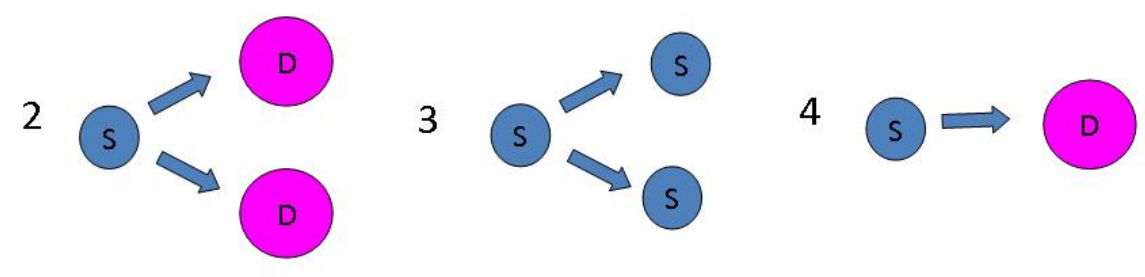

Figure 2: Possible modes of stem cell division.

other possible scenarios of cancer stem cells division are not completely excluded (see Fig. 2 ) the symmetric division giving rise to two identical daughter cells with stem cell properties; the symmetric division with two identical daughter cells with non-stem cell properties, and also the direct development of initial stem cell into non-stem one. All these scenarios are currently under hot discussion, as for normal, so for cancer stem cells. Non-stem (daughter) cells, as a rule undergo the symmetric division producing two identical daughter cells with non-stem cell properties. The question about other possible types of non-stem cell division, namely, the possibility for nonstem cancer cells to undergo under some specific conditions the process of non-stem to stem cell transition (analog of dedifferentiation for normal cell case), is one of the main open problems in current molecular biology (see Fig. 3 ). Considering the speed of cell divisions, it has been well documented that the speed of stem cells division are much slower than the speed of cell divisions of non-stem cells (see Fig. 4). The current theory for normal stem cells evolution is that stem cells remain undifferentiated due to environmental cues in their particular niche, and differentiate when they leave that niche or no longer receive corresponding signals (Whetton \& Graham, 1999). However, this theory still leaves open the main question: what is the nature of the signals for maintaining the proper population behavior of stem cells within the given niche? Thus, our mathematical model describing the main features of possible "instructive signals" maintaining the proper pattern of a system, can be valuable for addressing this important question not only for 
Usually suggested

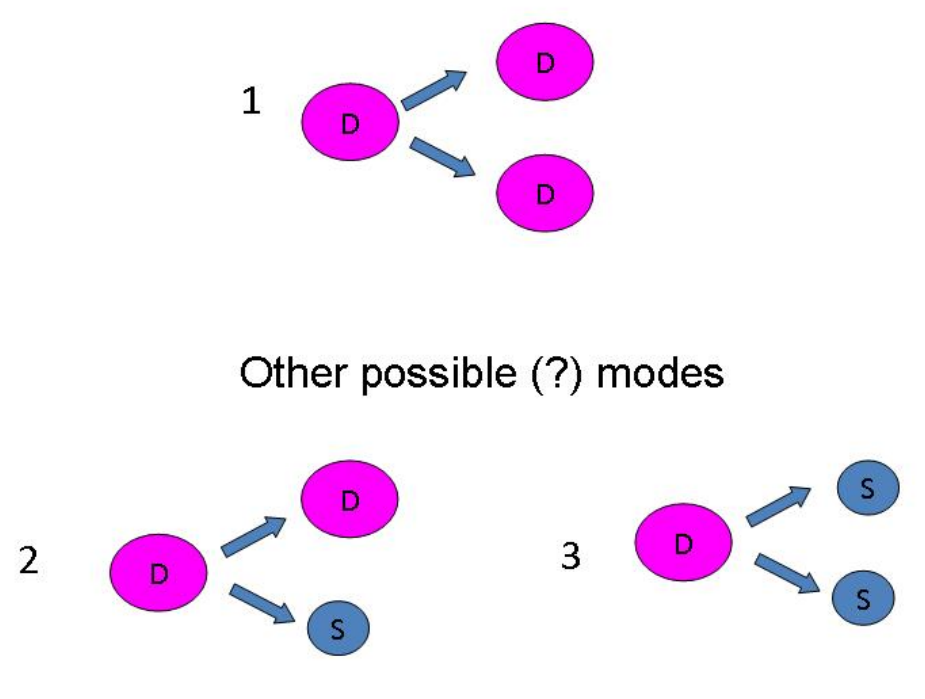

Figure 3: Possible modes of non-stem cell division.

cancer, but also for normal stem cells population behavior.

\section{The Concept Model}

We may then consider a mathematical model for the evolution of stem cells (S), and non-stem cells (D) populations. Based on the discussion above, we will consider that stem cells may divide either into two stem cells or into one stem cell and one non-stem cell, or into two non-stem cells, or into one non-stem cell (with a total rate $\lambda_{1}>0$ ), according to the following scheme (see Fig. 2)

$$
\begin{array}{llll}
S \Longrightarrow S & + & \text { with probability } & p_{1} \\
S \Longrightarrow D+ & D & \text { with probability } & p_{2} \\
S \Longrightarrow S+ & & & \\
S \Longrightarrow D & & \text { with probability } & p_{3} \\
S \Longrightarrow & \text { with probability } & p_{4}
\end{array}
$$




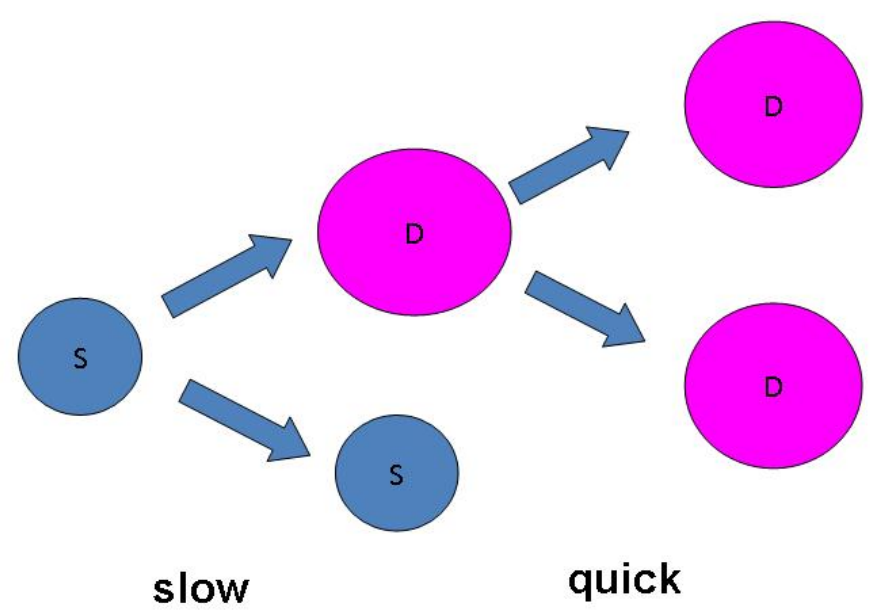

Figure 4: The speed of cell divisions.

such that

$$
p_{i} \geq 0, \quad i=1, \ldots, 4 ; \quad p_{1}+p_{2}+p_{3}+p_{4}=1 .
$$

We assume here that non-stem cells may divide either into two non-stem cells or into one stem cell and one non-stem cell (with a rate $\lambda_{2}$ )

$$
\begin{array}{lll}
D \Longrightarrow D+D & \text { with probability } & q_{1} \\
D \Longrightarrow S+D & \text { with probability } & q_{2}
\end{array}
$$

such that

$$
q_{j} \geq 0, \quad i=1,2 ; \quad q_{1}+q_{2}=1 .
$$

We have ignored the possible case 3 for non-stem cells as from Fig. 3. Its inclusion in the model would only lead to additional mathematical technicalities.

Stem cells may die at a rate $\gamma_{1}$, while non-stem cells may die at a rate $\gamma_{2}$.

We assume a time scale much larger than the typical cell cycle time and that cell populations are large enough to ignore randomness (Johnston et al. (2007)). We may then assume a one point 
(in space) model with a continuous time evolution, just to specify the main ingredients of the model of evolution of stem cells (S) and non-stem cells (D).

As a consequence, to start with, the mathematical model that we propose is given by the following system of ordinary differential equations.

$$
\begin{aligned}
\frac{d}{d t} S(t) & =\left(-\gamma_{1}+\left(-1+2 p_{3}+p_{1}\right) \lambda_{1}\right) S(t)+q_{2} \lambda_{2} D(t) \\
\frac{d}{d t} D(t) & =\left(p_{1}+2 p_{2}+p_{4}\right) \lambda_{1} S(t)+\left(-\gamma_{2}+q_{1} \lambda_{2}\right) D(t)
\end{aligned}
$$

subject to initial conditions $S(0)=S_{0}$, and $D(0)=D_{0}$, such that $\left(S_{0}, D_{0}\right) \in \mathbf{R}_{+}^{2},\left(S_{0}, D_{0}\right) \neq$ $(0,0)$.

System (2.7),(2.8) is an homogeneous system of two linear ODE equations whose matrix of (constant) coefficients is

$$
\mathbf{A}=\left(\begin{array}{cc}
\alpha_{S} & \beta_{D} \\
\beta_{S} & \alpha_{D}
\end{array}\right)
$$

where

$$
\begin{aligned}
& \alpha_{S}=-\gamma_{1}+\left(-1+2 p_{3}+p_{1}\right) \lambda_{1}, \quad \beta_{D}=q_{2} \lambda_{2} \\
& \beta_{S}=\left(p_{1}+2 p_{2}+p_{4}\right) \lambda_{1}, \quad \alpha_{D}=-\gamma_{2}+q_{1} \lambda_{2},
\end{aligned}
$$

with $\beta_{S} \geq 0$, and $\beta_{D} \geq 0$.

For the time being we assume that $\beta_{D}>0$, and $\beta_{S}>0$; under this circumstance, matrix $A$ has positive off-diagonal elements. Later the case $q_{2}=0$ will be analyzed in more detail.

Accordingly, an explicit solution of the ODE system (2.7), (2.8) is given by

$$
\begin{aligned}
S(t) & =C_{1} e^{r_{1} t}+C_{2} e^{r_{2} t} \\
D(t) & =\frac{1}{\beta_{D}}\left(S^{\prime}(t)-\alpha_{S} S(t)\right)
\end{aligned}
$$

where $r_{i}, i=1,2$ are the eigenvalues of matrix $A$, and the coefficients $C_{1}$, and $C_{2}$ are given by the initial conditions.

More specifically $r_{i}, i=1,2$ are the roots of the characteristic equation

$$
r^{2}-(\operatorname{Tr} \mathbf{A}) r+\operatorname{Det} \mathbf{A}=0
$$

where $\operatorname{Tr} \mathbf{A}=\alpha_{S}+\alpha_{D}$, Det $\mathbf{A}=\alpha_{S} \alpha_{D}-\beta_{S} \beta_{D} \neq 0$, and the discriminant is $\Delta=(\operatorname{Tr} \mathbf{A})^{2}-$ $4 \operatorname{Det} \mathbf{A}>0$. Therefore the two eigenvalues of $A$ are real; they are given by

$$
r_{1}=\frac{1}{2}\{\operatorname{Tr} \mathbf{A}-\sqrt{\Delta}\}, \quad r_{2}=r_{1}+\sqrt{\Delta}
$$


In conclusion, the solution of (2.7), (2.8) is:

$$
\begin{gathered}
S(t)=C_{1} e^{r_{1} t}+C_{2} e^{r_{2} t} \\
D(t)=\frac{1}{\beta_{D}}\left(C_{1}\left(r_{1}-\alpha_{S}\right) e^{r_{1} t}+C_{2}\left(r_{2}-\alpha_{S}\right) e^{r_{2} t}\right), t>0 .
\end{gathered}
$$

By remembering that $r_{2}=r_{1}+\sqrt{\Delta}$ it is easy to see that

$$
\frac{D(t)}{S(t)}=\frac{C_{1}\left(r_{1}-\alpha_{S}\right)+C_{2}\left(r_{2}-\alpha_{S}\right) e^{\sqrt{\Delta} t}}{\beta_{D}\left(C_{1}+C_{2} e^{\sqrt{\Delta} t}\right)}
$$

and therefore that for all initial conditions we have

$$
\frac{D(t)}{S(t)} \rightarrow \frac{r_{2}-\alpha_{S}}{\beta_{D}}>0 \quad \text { as } \quad t \rightarrow+\infty
$$

Since experimental data are expressed in terms of percentages of stem cells, as opposed to percentages of non-stem cells, with respect to the total number of cells

$$
N(t)=S(t)+D(t)
$$

we are interested in the evolution of the fractions

$$
s(t)=\frac{S(t)}{N(t)}, \quad d(t)=\frac{D(t)}{N(t)}
$$

such that

$$
s(t)+d(t)=1
$$

at any time $t \geq 0$.

From the limit (2.14) it is trivial to derive the corresponding limit for $s(t)$, as $t \rightarrow+\infty$.

2.0.1. Time evolution of $s(t)=\frac{S(t)}{N(t)}$, and its asymptotic behavior

In terms of fractions $s(t)$ and $d(t)$, system (2.7), (2.8) can be rewritten in the following form

$$
\begin{aligned}
\frac{d}{d t} s(t) & =P+Q s(t)+R s^{2}(t) \\
d(t) & =1-s(t), \quad t \geq 0
\end{aligned}
$$

subject to an initial condition $s(0) \in[0,1]$;

the above system is complemented by 


$$
\frac{d}{d t} N(t)=\left[-R s(t)+\left(\lambda_{2}-\gamma_{2}\right)\right] N(t)
$$

subject to an initial condition

$$
N(0)=N_{0}>0, \quad t \geq 0 .
$$

Here

$$
\begin{aligned}
P & =q_{2} \lambda_{2}, \\
Q & =\left(p_{3}-\left(p_{2}+p_{4}\right)\right) \lambda_{1}-\left(1+q_{2}\right) \lambda_{2}+\gamma_{2}-\gamma_{1}, \\
R & =\lambda_{2}-\left(1-p_{4}\right) \lambda_{1}-\left(\gamma_{2}-\gamma_{1}\right) .
\end{aligned}
$$

The differential equation (2.17) is a Riccati equation of the first type with constant coefficients, which can be explicitly solved (but we omit here its explicit solution). The main properties of system (2.17), (2.18) depend upon the function $f:[0,1] \rightarrow \mathbb{R}$, given by

$$
f(s):=P+Q s+R s^{2}, \quad s \in[0,1] .
$$

We may notice that, if we assume $q_{2}>0$, then

$$
\begin{gathered}
\left.\dot{s}(t)\right|_{s=0}=f(0)=q_{2} \lambda_{2}>0, \\
\left.\dot{s}(t)\right|_{s=1}=f(1)=\left(-1-p_{2}+p_{3}\right) \lambda_{1}<0,
\end{gathered}
$$

which imply that for any initial condition $s(0) \in[0,1]$, the corresponding solution of (2.17) is such that $s(t) \in(0,1)$ for all $t>0$.

The equilibria of the equation (2.17) are the roots of the algebraic equation

$$
f(s)=0, \quad s \in[0,1] .
$$

Because of (2.24), and (2.25), $f$ must admit at least one zero within $(0,1)$, and because of the structure of the function $f$, there exists exactly a unique root $s^{*} \in(0,1)$ of $(2.26)$ such that

$$
\dot{s}(t)=f(s)>0, \quad \text { for } \quad s \in\left[0, s^{*}\right), \quad \text { and } \quad \dot{s}(t)=f(s)<0, \text { for } \quad s \in\left(s^{*}, 1\right] .
$$

Of course no solution may cross the equilibrium $s^{*}$. Finally we may state the following result.

Theorem 1. A unique $s^{*} \in(0,1)$ exists such that $\left(s^{*}, d^{*}=1-s^{*}\right)$ is a globally asymptotically stable equilibrium for system (2.17), (2.18); specifically

$$
\left(s^{*}=\frac{\beta_{D}}{r_{2}+\beta_{D}-\alpha_{S}}, d^{*}=\frac{r_{2}-\alpha_{S}}{r_{2}+\beta_{D}-\alpha_{S}}\right) .
$$


The global stability of the nontrivial equilibrium $\left(s^{*}, d^{*}\right)$ can be derived via a simple Lyapunov function; its value derives from (2.14).

From all previous discussion it follows that the positivity of $s^{*} \in(0,1)$ is related to the positivity of $P=q_{2} \lambda_{2}=\beta_{D}$, i.e. to the positivity of the probability $q_{2}$ that normal cells $\mathrm{D}$ may divide into a stem cell $\mathrm{S}$ and a non-stem cell $\mathrm{D}$. If the probability of this elementary process is equal to zero, we see that also $s^{*}$ in Theorem 1 goes to zero. Therefore it becomes biologically interesting to see what happens when $q_{2}=0$, i.e. when it is not allowed non-stem cells to produce stem cells. However, before analyzing the case $q_{2}=0$ it can be useful to discuss more about the parameters.

\subsection{A discussion about the parameters, and the case $q_{2}=0$.}

Since the branching processes of non-stem cells (D) occur at a global rate faster than the ones of stem cells (S), it is reasonable to assume that $\lambda_{2}>\lambda_{1}$. Moreover, it seems reasonable to assume that the death rates $\gamma_{1}$, and $\gamma_{2}$ are about the same for both stem and non-stem cells, i.e. $\gamma_{1} \simeq \gamma_{2}$. Finally we additionally assume that the total branching rate $\lambda_{2}$ of non-stem cells exceeds the related death rate $\gamma_{2}$, i.e. $\lambda_{2}>\gamma_{2} \simeq \gamma_{1}$. By summarizing we assume

$$
\lambda_{2}>\lambda_{1} ; \quad \gamma_{1} \simeq \gamma_{2} ; \quad \gamma_{i}<\lambda_{2}, i=1,2 .
$$

The above assumptions (2.28) enable to define the sign of the quantities $P, Q, R$ defined by (2.20)-(2.22). Namely we have

$$
\begin{aligned}
& P=q_{2} \lambda_{2}>0, \\
& Q \simeq\left(p_{3}-\left(p_{2}+p_{4}\right)\right) \lambda_{1}-\left(1+q_{2}\right) \lambda_{2}<0, \\
& R \simeq \lambda_{2}-\left(1-p_{4}\right) \lambda_{1}>0
\end{aligned}
$$

whereas for the case $q_{2}=0$, we have

$$
\begin{aligned}
& P=0, \\
& Q \simeq\left(p_{3}-\left(p_{2}+p_{4}\right)\right) \lambda_{1}-\lambda_{2}<0, \\
& R \simeq \lambda_{2}-\left(1-p_{4}\right) \lambda_{1}>0 .
\end{aligned}
$$

We are now ready to discuss the case $q_{2}=0$; in this case $P=\beta_{D}=\lambda_{2} q_{2}=0$ which implies that the function $f$ defined in (2.23) now becomes

$$
f(s):=Q s+R s^{2}, \quad s \in[0,1] ;
$$

as a consequence

$$
\begin{aligned}
& \left.\dot{s}(t)\right|_{s=0}=f(0)=0 ; \\
& \left.\left.\dot{s}(t)\right|_{s=1}=f(1)=\left(-1-p_{2}+p_{3}\right)\right) \lambda_{1}<0 .
\end{aligned}
$$


Accordingly, one equilibrium is $\left(s^{*}=0, \quad d^{*}=1\right)$.

Since by (2.34) $R>0$, and by (2.37) $f(1)<0$, then

$$
f(s)=Q s+R s^{2}<0, \quad s \in(0,1],
$$

thus implying that no other equilibrium may exist in $(0,1]$. Further, since

$$
\frac{d}{d t} s(t)=f(s(t))<0 \quad \text { for all } \quad t>0 \quad \text { such that } \quad s(t) \in(0,1]
$$

we may claim the following.

Theorem 2. If $q_{2}=0$, the unique equilibrium $\left(s^{*}=0, d^{*}=1\right)$ is globally asymptotically stable in $[0,1] \times[0,1]$, and the convergence to the equilibrium is monotone.

Remark 3. We may conclude by observing that, if in the parameter values we drop the second and the third assumptions in (2.28), then it becomes possible, for the case $q_{2}=0$, to have $Q>0$, and $R<0$, maintaining true both (2.36), and (2.37). In this case, besides $\left(s^{*}=0, d^{*}=1\right)$, it must exist a nontrivial equilibrium too. We will not discuss this case further.

\subsection{About the total population.}

From (2.19) we know that

$$
N(t)=N_{0} \exp \left\{\int_{0}^{t}\left(-R s(\tau)+\lambda_{2}-\gamma_{2}\right) d \tau\right\} .
$$

If we take into account the case $\lambda_{2}>>\lambda_{1}$, and $\gamma_{2}<<\lambda_{2}$, we may use the approximation

$$
N(t) \gtrsim N_{0} \exp \left\{\int_{0}^{t}\left(-\lambda_{2} s(\tau)+\lambda_{2}\right) d \tau\right\}=N_{0} \exp \left\{\int_{0}^{t} \lambda_{2} d(\tau) d \tau\right\} .
$$

Because of the monotone convergence of the solutions to the equilibrium, it is not difficult to check that, in all cases,

$$
N(t) \uparrow+\infty, \quad \text { as } \quad t \rightarrow+\infty .
$$

The eventual explosion of the total population is not a surprise since we have not imposed any saturation (such as a logistic growth), since we may assume that the culture is continuously feeded by nutrients.

\section{Underlying field}

Again based on the introductory remarks, one may conjecture that, given constant proliferation rates $\left(\lambda_{1}\right.$ and $\left.\lambda_{2}\right)$, all proportions $p_{i}$ and $q_{j}$ of the elementary processes depend upon some underlying field $u$, (a biochemical signal) which is produced by the same population of cells 


$$
p_{i}=p_{i}(u), \quad i=1,2,3,4,
$$

such that

$$
\begin{gathered}
\sum_{i=1}^{4} p_{i}(u)=1, \quad \text { for any } \quad u \geq 0 \\
q_{j}=q_{j}(u), \quad i=1,2, \\
\sum_{j=1}^{2} q_{j}(u)=1, \quad \text { for any } \quad u \geq 0 .
\end{gathered}
$$

For an updated mathematical model, we introduce the functional response $g(S, D)$ for the production of the underlying field from existing stem cells and non-stem cells, and a decay rate $\varepsilon$ of the underlying field itself.

As far as the underlying field $u$ is concerned, we additionally consider the possibility that the response to existing cells may be delayed by a constant delay $r \geq 0$, so that the mathematical model finally becomes

$$
\begin{aligned}
\frac{d}{d t} u(t) & =-\varepsilon u(t)+g(S(t-r), D(t-r)) \\
\frac{d}{d t} S(t) & =-\gamma_{1} S(t)+\left(-1+2 p_{3}(u(t))+p_{1}(u(t))\right) \lambda_{1} S(t) \\
& +q_{2}(u(t)) \lambda_{2} D(t) \\
\frac{d}{d t} D(t) & =-\gamma_{2} D(t)+\left(-1+2 q_{1}(u(t))+q_{2}(u(t))\right) \lambda_{2} D(t) \\
& +\left(p_{1}(u(t))+2 p_{2}(u(t))+p_{4}(u(t))\right) \lambda_{1} S(t) .
\end{aligned}
$$

We may anticipate here that this extended model may now lead to a much larger variety of dynamical behaviors (see later in the section on simulations), so to stimulate further experiments under various scenarios.

For a simpler handling of the above system, we consider relative amounts, as for the case independent of $u$. Define

$$
s(t):=\frac{S(t)}{S(t)+D(t)}
$$

so that $s$ satisfies the equation

$$
s^{\prime}(t)=P(u(t))+Q(u(t)) s(t)+R(u(t)) s(t)^{2},
$$


with

$$
\begin{aligned}
& P(u)=q_{2}(u) \lambda_{2}, \\
& \left.Q(u)=\left(p_{3}(u)-\left(p_{2}(u)+p_{4}(u)\right)\right)\right) \lambda_{1}-\lambda_{2}-q_{2}(u) \lambda_{2}+\gamma_{2}-\gamma_{1}, \\
& R(u)=\lambda_{2}-\left(1-p_{4}(u)\right) \lambda_{1}-\left(\gamma_{2}-\gamma_{1}\right) .
\end{aligned}
$$

Note that Equation (3.4) depends on $p_{4}, q_{2}$ and only on the combination $\left(p_{3}-\left(p_{2}+p_{4}\right)\right)$.

For a technical simplification let us assume that

$$
g(S(t), D(t))=g\left(\frac{S(t)}{S(t)+D(t)}\right)
$$

so that we may take

$$
g(S(t), D(t))=g(s(t))
$$

with some function $g(s)$.

Based on previous assumptions, if we let $r \in \mathbb{R}_{+}$be the constant delay, we take the mathematical model as follows.

$$
\begin{aligned}
\frac{d}{d t} u(t) & =-\varepsilon u(t)+g(s(t-r)) \\
\frac{d}{d t} s(t) & =f(u(t), s(t)) \\
d(t) & =1-s(t),
\end{aligned}
$$

for $t \geq 0$. complemented by the equation for the total population

$$
N^{\prime}(t)=\left[-R(u(t)) s(t)+\lambda_{2}-\gamma_{2}\right] N(t)
$$

and subject to suitable initial conditions

$$
(u(\cdot), s(\cdot)) \in C_{b}\left([-r, 0], \mathbb{R}_{+} \times[0,1]\right) .
$$

Here

$$
f(u, s):=P(u)+Q(u) s+R(u) s^{2} .
$$

About the structure of the system we assume that

(i) $\varepsilon>0$, a constant;

(ii) $\lambda_{i}, \gamma_{i} \in \mathbb{R}_{+}, \quad i=1,2$, satisfy the assumptions in (2.28);

(iii) $g:[0,1] \rightarrow \mathbb{R}_{+}, \quad g \in C^{1}((0,1))$; with $g^{\prime}(s)>0$ for any $s \in(0,1)$; 
(iv) $p_{i}: \mathbb{R}_{+} \rightarrow(0,1), \quad p_{i} \in C^{1}\left(\mathbb{R}_{+}\right), \quad i=1,2,3,4$,

such that $\sum_{i=1}^{4} p_{i}(u)=1$, for any $u \in \mathbb{R}_{+}$;

(v) $q_{j}: \mathbb{R}_{+} \rightarrow(0,1), \quad q_{j} \in C^{1}\left(\mathbb{R}_{+}\right), \quad j=1,2$,

such that $\sum_{j=1}^{2} q_{j}(u)=1$, for any $u \in \mathbb{R}_{+}$;

Under the assumptions (ii), (iv), (v) we have that

$$
\begin{aligned}
& f(u, 0)=P(u)=q_{2}(u) \lambda_{2}>0, \\
& f(u, 1)=P(u)+Q(u)+R(u)=\left(-1-p_{2}(u)+p_{3}(u)\right) \lambda_{1}<0,
\end{aligned}
$$

for all $u>0$.

Furthermore, the assumptions (ii), (iv), (v) also imply that

$$
\begin{aligned}
Q(u) & \left.=\left(p_{3}(u)-\left(p_{2}(u)+p_{4}(u)\right)\right)\right) \lambda_{1}-\lambda_{2}-q_{2}(u) \lambda_{2}+\gamma_{2}-\gamma_{1} \leq \\
& \leq p_{3}(u) \lambda_{1}-\lambda_{2}<0 ; \\
R(u) & =\lambda_{2}-\left(1-p_{4}(u)\right) \lambda_{1}-\left(\gamma_{2}-\gamma_{1}\right) \geq \lambda_{2}-\lambda_{1}>0
\end{aligned}
$$

for all $u>0$, that is the signs of $P(u), Q(u)$, and $R(u)$ remain constant for all $u>0$.

On the other hand, we wish that the underlying field $u$ has to provide a delayed feedback on the observed percentage level of stem cells; i.e. an increase of $s(t-r)$ ) has to imply an increase of $u(t)$ thanks to Equation (3.9). Such an increase of $u(t)$ must imply a decrease of $s(t)$ via Equation (3.10). This can be obtained by imposing the following additional assumption.

(vi) $f \in C^{1,1}\left(\mathbb{R}_{+}-\{0\} \times(0,1)\right)$, must satisfy

$$
\frac{\partial f(u, s)}{\partial u}<0 \quad \text { for all } \quad(u, s) \in \mathbf{R}_{+} \times(0,1) .
$$

\subsection{Remarks}

(P.1) Properties (3.14) and (3.15) ensure that $s(t) \in(0,1)$, for all $t>0$.

(P.2) The positivity of $g(s)$, for all $s \in(0,1)$, implies that $u(t)>0$, for all initial conditions $u(0) \geq 0$.

(P.3) Since $s(t) \in(0,1)$, for all $t>0$, and $g(s)$ is strictly increasing with $s$, we know that

$$
g(s(t))<g(1)
$$

and therefore, from (3.9) we get 


$$
\begin{aligned}
\frac{d}{d t} u(t) & <-\varepsilon u(t)+g(1), \\
\limsup _{t \rightarrow+\infty} u(t) & \leq \frac{g(1)}{\varepsilon} .
\end{aligned}
$$

(P.4) Thanks to (P.1)-(P.3) we may claim that the compact set

$$
\Omega:=\left[0, \frac{g(1)}{\varepsilon}\right] \times[0,1]
$$

is a global attractor and a positively invariant set for the solution of system (3.9)-(3.10); moreover any solution staying on the boundary of $\Omega$ will eventually enter into the interior of $\Omega$.

The equilibria of system (3.9)-(3.10) are independent of the delay value $r$, so that we may study the equilibria of the ODE system associated to (3.9)-(3.10) when $r=0$, for which the compact set $\Omega$ is still an invariant set. By the Poincaré-Bendixon Theorem and the Bendixon Criterion (see e.g. [15], page 44) applied to the planar flow of the ODE system, we may claim the following.

Proposition 4. If in system (3.9)-(3.10) we choose

$$
\varepsilon>\sup _{(u, s) \in \Omega}(2 R(u) s+Q(u))
$$

then, for any choice of $r \geq 0$ there exists at least one (positive) equilibrium $\left(u^{*}, s^{*}\right)$ of system (3.9)-(3.10), in $\stackrel{\circ}{\Omega}$.

As far as the stability of such an equilibrium is concerned, for the case $r=0$ we proceed as follows.

By defining

$$
A\left(u^{*}, s^{*}\right)=\left.\frac{\partial f(u, s)}{\partial u}\right|_{\left(u^{*}, s^{*}\right)} ; \quad B\left(u^{*}, s^{*}\right)=-\left.\frac{\partial f(u, s)}{\partial s}\right|_{\left(u^{*}, s^{*}\right)},
$$

the Jacobi matrix of system (3.9)-(3.10) for $r=0$ (no delay) at $\left(u^{*}, s^{*}\right)$ is given by

$$
J=\left[\begin{array}{cc}
-\varepsilon & g^{\prime}(s *) \\
A\left(u^{*}, s^{*}\right) & -B\left(u^{*}, s^{*}\right)
\end{array}\right]
$$

As a consequence of known results, we may then state that necessary and sufficient conditions for the (local) asymptotic stability of the equilibrium $\left(u^{*}, s^{*}\right)$ are

$$
\varepsilon+B\left(u^{*}, s^{*}\right)>0 \quad \text { and } \varepsilon B\left(u^{*}, s^{*}\right)-g^{\prime}\left(s^{*}\right) A\left(u^{*}, s^{*}\right)>0 .
$$


Since the equilibrium $s^{*}$ is the smallest root of $P\left(u^{*}\right)+Q\left(u^{*}\right) s^{*}+R\left(u^{*}\right) s^{* 2}=0$, because of the negativity of $Q(u)$ we obtain

$$
s^{*}<\frac{1}{2} \frac{\left|Q\left(u^{*}\right)\right|}{R\left(u^{*}\right)} .
$$

Accordingly $-B\left(u^{*}, s^{*}\right)=Q\left(u^{*}\right)+2 R\left(u^{*}\right) s^{*}<0$, implying $B\left(u^{*}, s^{*}\right)>0$.

Since by (i) we have $g^{\prime}\left(s^{*}\right) A\left(u^{*}, s^{*}\right)<0$, it is clear that conditions (3.24) hold true whenever a positive equilibrium $\left(u^{*}, s^{*}\right)$ exists. Hence the following holds.

Proposition 5. If in system (3.9)-(3.10) we choose $\varepsilon$ satisfying (3.22) then, for $r=0$ there exists one (positive) equilibrium $\left(u^{*}, s^{*}\right)$ of system (3.9)-(3.10), in $\stackrel{\circ}{\Omega}$, which is asymptotically stable.

\subsection{Delay induced Hopf bifurcation.}

The problem we are considering in this section is the following one; in the delay equations (3.9)(3.10) let us choose the delay $r$ as bifurcation parameter.

According to Proposition 5, the equilibrium $X^{*}=\left(u^{*}, s^{*}\right)^{T}$ is locally asymptotically stable, that is $\operatorname{Re} \lambda<0$, for all the characteristic roots $\lambda$ of the Jacobi matrix $J$.

We now investigate the possible occurrence of delay induced stability switches at the equilibrium $X^{*}$ by increasing the delay from the value $r=0$.

Let us denote by $X(t)=(u(t), s(t))^{T}$, and by $X_{t}:=(u(t+\theta), s(t+\theta))^{T}, \theta \in[-r, 0]$.

Accordingly the delay equations (3.9)-(3.10) become

$$
\frac{d}{d t} X(t)=G\left(X_{t} ; r\right)
$$

subject to an initial condition $\phi:=X_{t=0}=\left(\phi_{1}=u(\theta), \phi_{2}=s(\theta)\right)^{T}, \theta \in[-r, 0]$, with $\phi \in C:=$ $C\left([-r, 0], \mathbf{R}^{2}\right)$.

In (3.25) $G: C \rightarrow \mathbf{R}^{2}$ denotes the vector function

$$
G\left(X_{t} ; r\right)=\left(\begin{array}{l}
-\varepsilon u(t)+g(s(t-r)) \\
f(u(t), s(t))
\end{array}\right)
$$

with $G\left(X^{*} ; r\right)=0$ for all $r \geq 0$.

As a first step we linearize Equation (3.25); we first translate $X^{*}$ into $x^{*}=0$, by the transformation $X(t)=X^{*}+x(t)$, so that Equation (3.25) becomes

$$
\frac{d}{d t} x(t)=F\left(x_{t} ; r\right)
$$

subject to the initial condition $\phi \in C$; where now $F: C \rightarrow \mathbf{R}^{2}$ is the corresponding transform of $G$ such that $F(0 ; r)=0$ for all $r \geq 0$.

The linearization of the function $F$ around $x=0$ is obtained as (see e.g. [30], Sect. 4.6)

$$
F\left(x_{t} ; r\right)=L(r) x_{t}+R\left(x_{t} ; r\right)
$$


such that $L(r): C \rightarrow \mathbf{R}^{2}$ is a bounded linear operator, and

$$
\lim _{\psi \rightarrow 0} \frac{\|R(\psi ; r)\|}{\|\psi\|}=0, \quad \text { for all } \quad r \geq 0 .
$$

It is easy to realize that the linear operator $L(r)$ is such that

$$
L(r) x_{t}=J_{x} x(t)+J_{r} x(t-r)
$$

where

$$
J_{x}=\left(\begin{array}{cc}
-\varepsilon & 0 \\
A\left(u^{*}, s^{*}\right) & -B\left(u^{*}, s^{*}\right)
\end{array}\right)
$$

and

$$
J_{r}=\left(\begin{array}{cc}
0 & g^{\prime}\left(s^{*}\right) \\
0 & 0
\end{array}\right) .
$$

The characteristic equation associated with the linear operator to be analyzed is thus

$$
\operatorname{det}\left[J_{x}+\exp (-\lambda r) J_{r}-\lambda I\right]=\operatorname{det}\left[\begin{array}{cc}
-\varepsilon-\lambda & g^{\prime}\left(s^{*}\right) \exp (-\lambda r) \\
A\left(u^{*}, s^{*}\right) & -B\left(u^{*}, s^{*}\right)-\lambda
\end{array}\right]=0 .
$$

for $\lambda \in \mathbb{C}$.

Simple computations show that Equation (3.32) can be rewritten as

$$
\lambda^{2}+a \lambda+c+d e^{-\lambda r}=0
$$

with coefficients

$$
\begin{gathered}
a=\varepsilon+B\left(u^{*}, s^{*}\right) \\
b=\varepsilon B\left(u^{*}, s^{*}\right), \\
c=-g^{\prime}\left(s^{*}\right) A\left(u^{*}, s^{*}\right) .
\end{gathered}
$$

In the following we apply the Hopf bifurcation Theorem as from [30] (see Theor.4.8. p.55, and references therein) (the solution $\lambda(r)$ of (3.33) is parameterized in terms of $r \geq 0$ ).

Theorem 6. (Hopf bifurcation) Assume that there exists a bifurcation value $r_{0}$ at which the characteristic equation admits a pair of simple imaginary roots $\pm i \omega_{0}$, with $\omega_{0}>0$, and no other root is an integer multiple of $i \omega_{0}$. Further assume that at $r_{0}$ the transversality condition $\left.\frac{d \operatorname{Re}(\lambda(r))}{d r}\right|_{r_{0}}>0$ holds.

Then there exist real valued even functions $r(\epsilon)$ and $T(\epsilon)$ satisfying $r(0)=r_{0}$ and $T(0)=\frac{2 \pi}{\omega_{0}}$, and a non-constant $T(\epsilon)$-periodic function $x(t, \epsilon)$, with all functions being continuously differentiable functions in $\epsilon$ in a sufficiently small neighborhood of $\epsilon=0$, such that $x_{\epsilon}(t)$ is a solution of (3.27) and $x_{\epsilon}(t)=\epsilon y_{\epsilon}(t)$ where $y_{0}$ is a $\frac{2 \pi}{\omega_{0}}$-periodic solution of $\dot{y}(t)=L\left(r_{0}\right) y_{t}$. 
Here we omit the question about the stability of the bifurcating periodic solutions. Searching for the value of the delay at which the equilibrium $X^{*}$ switches from asymptotic stability to instability, by an increasing delay, we follow [11].

The inequalities (3.24), which hold true for all delays $r \geq 0$, imply that in the characteristic equation the coefficients must satisfy

$$
a>0, \quad c+d>0, \quad \text { for all } \quad r \geq 0 .
$$

Inequalities (3.37) imply that for any delay $r \geq 0, \lambda=0$ cannot be a root of (3.33); hence, a stability switch from asymptotic stability to instability can only occur if at some delay value $r_{0}$ a pair of simple imaginary roots $\pm i \omega, \quad \omega \in \mathbf{R}_{+}$, cross the imaginary axis towards $\mathbf{C}^{+}$(i.e. the right hand side of the complex plane $\mathbf{C}$ ).

According to the Hopf bifurcation Theorem, the stability switch value $r_{0}$ too is a bifurcation value of the delay.

Easy computations show that

(i) if $c^{2}-d^{2} \geq 0$, then there are no positive real solutions for $\omega$, i.e. the equilibrium $\left(u^{*}, s^{*}\right)$ remains asymptotically stable for all delays $r>0$.

(ii) if $c^{2}-d^{2}<0$, then there is one real positive root for $\omega$, say $\omega_{+}$, given by

$$
\omega_{+}=\left\{\frac{1}{2}\left[\left(2 c-a^{2}\right)+\sqrt{\Delta}\right]\right\}^{\frac{1}{2}}
$$

where

$$
2 c-a^{2}=-\left(\varepsilon^{2}+B\left(u^{*}, s^{*}\right)^{2}\right)<0,
$$

and

$$
\Delta:=\left(2 c-a^{2}\right)^{2}-4\left(c^{2}-d^{2}\right)>0 .
$$

The pair of characteristic roots $\lambda= \pm i \omega_{+}, \omega_{+} \in \mathbb{R}_{+}$cross the imaginary axis according to

$$
\operatorname{sign}\left\{\left(\frac{d(\operatorname{Re}(\lambda))}{d r}\right)_{\lambda= \pm i \omega_{+}}\right\}=\operatorname{sign}\left\{a^{2}-2 c+2 \omega_{+}^{2}\right\}=\operatorname{sign}\{\sqrt{\Delta}\},
$$

i.e. towards $\mathbb{C}^{+}$. The values of the delay at which such a crossing of the imaginary axis occurs are given by

$$
r_{n}=\frac{\theta+2 n \pi}{\omega_{+}}, n \in \mathbb{N}
$$

where $\theta \in[0,2 \pi)$ is the solution of the following system 


$$
\sin \theta=\frac{a \omega_{+}}{d}, \quad \cos \theta=\frac{\omega_{+}^{2}-c}{d} .
$$

Since $c+d>0$, the occurrence (or not) of stability switches depends on the sign of

$$
c-d=\varepsilon B\left(u^{*}, s^{*}\right)+g^{\prime}\left(s^{*}\right) A\left(u^{*}, s^{*}\right) .
$$

By increasing the delay $r$, the "stability switch" may only occur at the smallest of the values $r_{n}$ for which the characteristic equation (3.33) admits solutions. Further, according to the Hopf bifurcation Theorem, we have the following

Theorem 7. (i) If $c-d \geq 0$, the nontrivial equilibrium $X^{*}=\left(u^{*}, s^{*}\right)$ is (locally) asymptotically stable for any delay $r \geq 0$.

(ii) If $c-d<0$, there exists a delay $r_{0}=\frac{\theta}{\omega_{+}}>0$ such that the nontrivial equilibrium $X^{*}=$ $\left(u^{*}, s^{*}\right)$ is (locally) asymptotically stable for any delay $0 \leq r<r_{0}$, unstable for $r>r_{0}$, and the delay system (3.25) admits periodic solutions, the period of which is $T(r) \simeq \frac{2 \pi}{\omega_{+}}$, for $r$ sufficiently close to $r_{0}$, and an Hopf bifurcation towards instability takes place at $r=r_{0}$.

Note that a necessary condition for $c-d<0$ to hold is that $g^{\prime}\left(s^{*}\right) A\left(u^{*}, s^{*}\right)<0$, as already assumed in (i) above.

Remark 8. In case (ii), due to continuous dependence of the solution upon the delay parameter $r$, we may expect that for $r<r_{0}$, but sufficiently close to $r_{0}$, stability of the nontrivial equilibrium may occur via damped oscillations.

It may be of interest to notice that a similar behaviour has been obtained for the dynamical behaviour of a population of stem-nonstem cells in [36], by admitting a different kind of model for cell-cell communication.

\section{Simulations}

In the simulations, as far as the relevant parameters are concerned, we have chosen values in accordance with the usual biological assumptions (2.28), i.e.

$$
\lambda_{1}=1, \quad \lambda_{2}=3 ; \quad \gamma_{1}=\gamma_{2}=0.1
$$

As a possible dependence of the parameters $p_{i}$ and $q_{j}$ upon the underlying field $u$ we take the logistic one; we assume

$$
\begin{gathered}
p_{k}(u)=p_{k k} h_{1}(u), \quad k=1,2,3, \\
p_{4}(u)=1-\left(p_{3}(u)+p_{1}(u)+p_{3}(u)\right) ; \\
p_{11}=0.75, \quad p_{22}=0.5, \quad p_{33}=1 .
\end{gathered}
$$


Moreover,

$$
\begin{gathered}
q_{1}(u)=q_{11} h_{2}(u), \\
q_{2}(u)=1-q_{1}(u),
\end{gathered}
$$

with

$$
q_{11}=1
$$

where

$$
h_{1}(u)=\frac{p p_{0}}{\left(p-p_{0}\right) e^{-\alpha_{p} u}+p_{0}}, \quad p=0.4, \quad p_{0}=0.1, \quad \alpha_{p}=1
$$

and

$$
h_{2}(u)=\frac{q q_{0}}{\left(q-q_{0}\right) e^{-\alpha_{q} u}+q_{0}}, \quad q=0.7, \quad q_{0}=0.7, \quad \alpha_{q}=2 .
$$

respectively, for a given set of parameters.

In this way the parameter $p_{k}(u)$ may range from $p_{k k} p_{0}$ to $p_{k k} p, \quad k=1,2,3$; similarly the parameter $q_{1}(u)$ may range from $q_{11} q_{0}$ to $q_{11} q$. The specific values of $p, p_{0}, \alpha_{p}$, and $q, q_{0}, \alpha_{q}$, have been arbitrarily chosen for the time being; as for the many free other parameters, they will be the subject of an inverse problem, once reliable data will be available.

The other relevant parameter $\varepsilon$ has been chosen so to guarantee the existence of a nontrivial equilibrium $\left(u^{*}, s^{*}\right)$ of the delay differential system (3.9)-(3.10).If we take, as an example, $u^{*}=$ 0.5 , from the second equation we get $s^{*}=0.38056$; hence

$$
\varepsilon=\frac{g\left(s^{*}\right)}{u^{*}}
$$

The last element to be assigned is the function $g$ in (3.9); as a very arbitrary choice we have taken $g(s)=\exp (w s)$, with $w \in \mathbb{R}_{+}$, to be chosen later in order to guarantee a possible Hopf bifurcation.

As a consequence of the above choices,

$$
A\left(u^{*}, s^{*}\right)=-0.50835 ; \quad B\left(u^{*}, s^{*}\right)=3.16015,
$$

so that the equilibrium $\left(u^{*}, s^{*}\right)$ is asymptotically stable when $r=0$.

The condition of stability switch for an increasing delay $r$ becomes

$$
c-d=\exp \left(w s^{*}\right)\left\{\frac{B\left(u^{*}, s^{*}\right)}{u^{*}}-w\left|A\left(u^{*}, s^{*}\right)\right|\right\}<0,
$$

that is

$$
w>w_{c}:=\frac{B\left(u^{*}, s^{*}\right)}{u^{*}\left|A\left(u^{*}, s^{*}\right)\right|}=12.4329 .
$$

It is therefore sufficient to choose, for example $w=13$ ti imply $c-d<0$. 

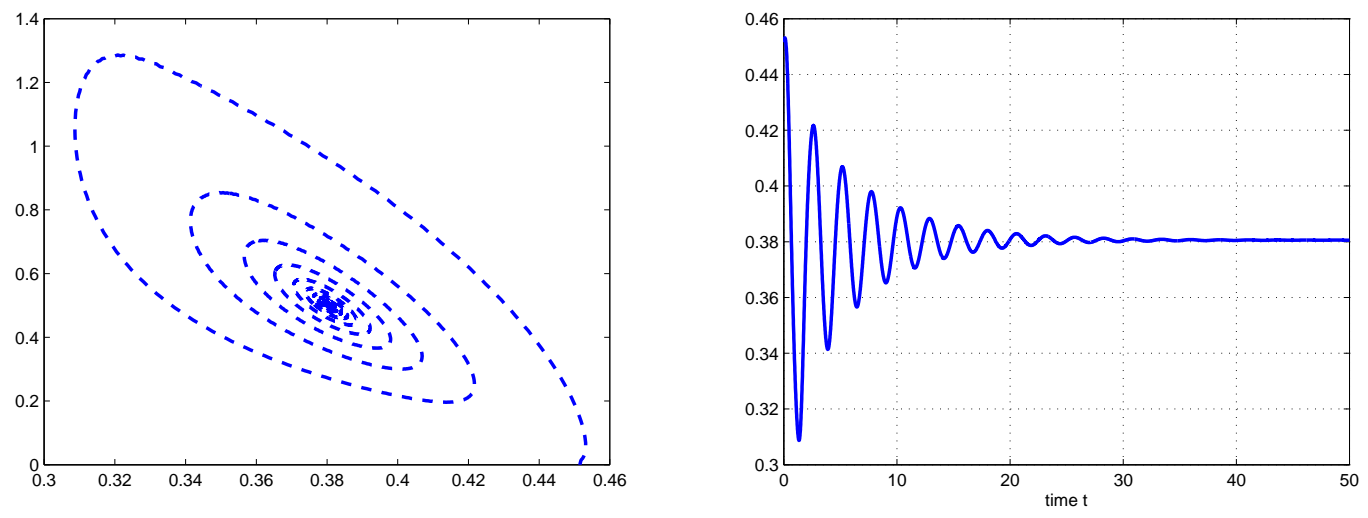

Figure 5: $r=1$. Damped oscillations can be observed as from the theory.
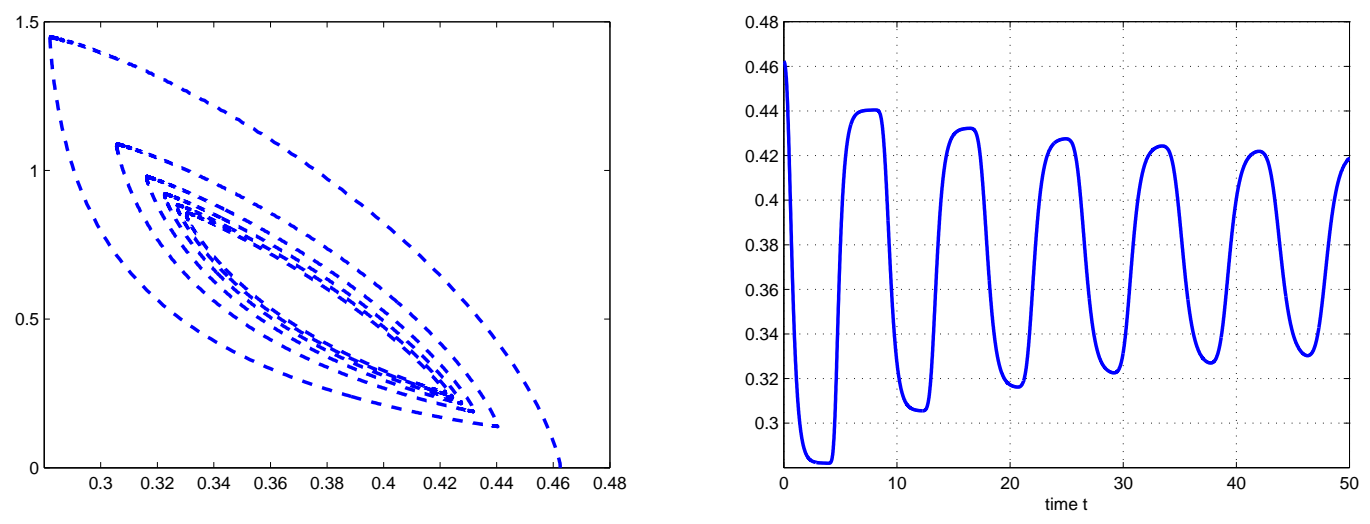

Figure 6: $r=4$. Convergence to sustained oscillations can be observed as from the theory.

With this choice we get $\varepsilon=\frac{\exp (13 * 0.38056)}{0.5}=281.5829$, and with the parameters chosen as above, we get that, for increasing $r$, the first stability switch occurs at $r_{0}$ given by

$$
r_{0}=\frac{\theta}{\omega_{*}}=3.093
$$

In accordance with Theorem 7 (ii), we may expect that, for any delay $r<r_{0}$, we observe damped oscillations, while for $r>r_{0}$ sustained oscillations occur.

In Figure 5 we have taken $r=1<3.093$, while in Figure 6 we have taken $r=4>3.093$. Both numerical simulations confirm our theoretical results.

The delay differential system (3.9) - (3.10) has been initialized by the history during the time interval $[-r, 0]$, obtained by imposing $u(\tau)=0$, for any $\tau \in[-r, 0]$, and solving equation (3.10) with $s(-r)=0.60$. 


\section{Conclusions}

The main motivation of this research was to establish a mathematical model which might explain an intriguing experimental fact, i.e. that the percentage of cancer stem cell population is maintained at the same level during many years, and also, that this percentage starting from around 100 percent in isolated population, rapidly decreases and finally stabilizes at the same characteristic level for this given cell line. To address the question about cancer stem cell population stability, we have proposed a frame ODE model based on a catalogue of possible cell divisions of stem and non-stem cells, and have extended our model so to include an additional variable, as a mathematical formalization of cell-cell communications in terms of a possible physical entity that we call underlying field. Next, as far as the underlying field was concerned, we additionally considered the possibility that a realistic response of this field to existing cells may be delayed by a constant time delay.

An assumption of regulation of tissue structure by an underlying (morphogenetic) field is a crucial point in biology since the concept of "positional information" was suggested by Wolpert (1969), and the mathematical evidence of this hypothesis is extremely important, especially for problems of pattern formation; indeed in a spatially structured model the proposed underlying signal may carry additional information regarding specific locations in space, thus driving specific behaviors accordingly. The underlying field concept, provided here, allows to give a new insight into the problem of cell-cell communication issue influencing the dynamics of population behavior of stem and non-stem cells. We may note that the description of the behavior of this variable $(u)$ corresponding to the underlying field, which we provide here, may help to elucidate a specific biochemical nature of a substance(s) responsible for such a field formation.

From a mathematical point of view the inclusion of the third variable $(u)$ in the equations describing the dynamics of cell populations, allows a larger variety of possible dynamical behaviors, including the possibility of an oscillatory behavior in the time evolution of stem cells, either with damped or with standing oscillations, as predicted in Zhdanov (2007) too. We wish to notice that the stability of a characteristic percentage level of stem cells in this case can still be proven, but only for sufficiently small delays in the response of the underlying field to modifications in the cells concentrations.

Also, our modelling helps to address a very important question in current molecular biology, which is under a hot discussion and is still open: is it possible for cancer non-stem cells under some specific conditions to undergo cell division producing cancer stem cells? All experiments reporting this possibility, were considered to be proved not well enough, or to be doubted due to the specific details of stem cells markers used for these investigations.

Here we have shown a set of realistic assumptions on the parameters under which the stability of cancer stem cells population level is possible only provided $q_{2}>0$ (meaning the process of nonstem to stem cell transition ). This may be considered as an additional tool for experimentalists for solving this question. It is very important to note that a significant advantage of the presented model is that theoretically it may be applicable to any cell population dynamics driven by stem cells. It comes from the already mentioned fact that the two main characteristics, which we put as a basic ones for our modelling, are the same for both normal and cancer stem cells; namely 
asymmetric divisions with relatively slow rate for stem cells, versus symmetric divisions with relatively quick rate for non-stem cells; and the tendency of maintaining the proper percentage of stem cells in the whole tissue (culture). Thus, generally speaking, our mathematical model, describing the main features of the "instructive signals" maintaining the proper pattern of a system, can be applicable also to normal stem cells population behavior. For this (normal) cases "non-stem cells" in our model should be understood as differentiated cells of normal tissues. However, due to the fact that the experimental observations on which we based our model, were obtained on cancer cell cultures (because non-stem cells cannot be cultivated in cell cultures without losing their properties), we have to be accurate to consider a cancer stem cells behavior as the central problem of the paper. This explains our main emphasis on the modelling of cancer stem cell population behavior. In conclusion, we may say that available experimental data at the moment are not decisive to validate this or that model, but seem to be in contrast with simple models which do not include communication among cells. On the other hand, existing experimental knowledge has suggested the model that we present here; our hope is that in turn our model may suggest new experiments to validate the assumptions on which we have based the model itself. As a matter of example, a crucial experiment would be to validate the existence of an oscillatory behavior in the time evolution of stem cells, either with damped or with standing oscillations.

Aknowledgements. We are grateful to Annick Harel-Bellan for inspiring and fruitful discussions on the biological content of the paper. Thanks are due to Stefan Kindermann (University of Linz, Austria) for relevant discussions on its mathematical aspects. The research contribution by Beretta and Capasso has been performed within the Italian PRIN project "Mathematical Theory of Populations: Methods, Models, Comparison with Experimental Data" (grant 2007.77BWEP-003). The research contribution by Morozova has been performed within the project "Cancérôpole" Île-de-France, n. 2007-1-ACI-CNRS EST-1".

We wish to thank the anonymous Referees too, for their valuable contribution to the improvement of the paper.

\section{References}

[1] M. Al-Hajj, M.S. Wicha, A. Benito-Hernandez, S.J. Morrison, M.F. Clarke. Prospective identification of tumorigenic breast cancer cells. Proc. Natl Acad. Sci. USA, 100 (2003), 3983-3988.

[2] S. Bao, Q. Wu, R.E. McLendon, Y. Hao, Q. Shi, A.B. Hjelmeland, M.W. Dewhirst, D.D. Bigner, J.N. Rich. Glioma stem cells promote radioresistance by preferential activation of the DNA damage response. Nature, 444 (2006), 756-760. 
[3] B. Barrilleaux, D.G. Phinney, D.J. Prockop, K.C. O'Connor. Review: ex vivo engineering of living tissues with adult stem cells. Tissue Eng., 12 (2006), 3007-3019.

[4] D. Bonnet, J.E. Dick. Human acute myeloid leukemia is organized as a hierarchy that originates from a primitive hematopoietic cell. Nat. Med., 3 (1997), 730-737.

[5] M.F. Clarke, J.E. Dick, P.B. Dirks, C.J. Eaves, C.H. Jamieson, D.L. Jones, J. Visvader, I.L. Weissman, G.M. Wahl. Cancer stem cells-Perspectives on current status and future directions: AACR workshop on cancer stem cells. Cancer Res., 66 (2006), 9339-9344.

[6] M. Dean, T. Fojo, S. Bates. Tumour stem cells and drug resistance. Nat. Rev. Cancer, 5 (2005), 275-284.

[7] M. Diehn, M.F. Clarke. Cancer stem cells and radiotherapy: new insights into tumor radioresistance. J. Natl. Cancer Inst., 98 (2006), 1755-1757.

[8] G. Dontu, W.M. Abdallah, J.M. Foley, K.W. Jackson, M.F. Clarke, M.J. Kawamura, M.S. Wicha. In vitro propagation and transcriptionalprofiling of human mammary stem/progenitor cells. Genes Dev., 17 (2003), 1253-1270.

[9] A. D'Onofrio, I.P.M. Tomlison. A nonlinear mathematical model of cell renewal, turnover and tumorigenesys in colon crypts. J. Theor. Biol., 244 (2007), 367-374.

[10] C.E. Eyler, J.N. Rich. Survival of the fittest: cancer stem cells in therapeutic resistance and angiogenesis. J. Clin. Oncol., 26 (2008), 2839-2845.

[11] H.I. Freedman, Y. Kuang. Stability switches in linear scalar neutral delay equations. Funkcial. Ekvac., 34 (1991), 187-209.

[12] R.L. Gardner. Stem cells: potency, plasticity and public perception. J. Anat., 200 (2002), 277-282.

[13] J.M. Gimble, A.J. Katz, B.A. Bunnell. Adipose-derived stem cells for regenerative medicine. Circ. Res., 100 (2007), 1249-1260.

[14] C. Ginestier, M.S. Wicha. Mammary stem cell number as a determinate of breast cancer risk. Breast Cancer Res., 9 (2007), 109.

[15] J. Guckenheimer, Ph. Holmes. Nonlinear oscillations, dynamical systems, and bifurcation of vector fields. Springer-Verlag, New York, 1983.

[16] P.B. Gupta,T.T. Onder, G. Jiang, K. Tao, C. Kuperwasser, R.A. Weinberg, E.S. Lander. Identification of selective inhibitors of cancer stem cells by high-throughput screening. Cell, 138 (2009), 645-659.

[17] M.D. Johnston, C.M. Edwards, W.F. Bodmer, P.K. Maini, S.J. Chapman. Mathematical modelling of cell population dynamics in the colonic crypt and in colorectal cancer. PNAS, 104 (2007), 4008-4013. 
[18] S.H. Lang, F. Frame, A. Collins, Prostate cancer stem cells. J. Pathol., 217 (2009), 299-306.

[19] C. Li, D.G. Heidt, P. Dalerba, C.F. Burant, L. Zhang, V. Adsay, M. Wicha, M.F. Clarke, D.M. Simeone. Identification of pancreatic cancer stem cells. Cancer Res. , 67 (2007), 10301037.

[20] X. Li, M.T. Lewis, J. Huang, C. Gutierrez, C.K. Osborne, M.F. Wu, S.G. Hilsenbeck, A. Pavlick, X. Zhang, G.C. Chamness, et al. Intrinsic resistance of tumorigenic breast cancer cells to chemotherapy. J. Natl. Cancer Inst., 100 (2008), 672-679.

[21] N.J. Maitland, A.T. Collins. Prostate cancer stem cells: a new target for therapy. J. Clin. Oncol., 26 (2008), 2862-2870.

[22] S.A. Mani, W. Guo, M.J. Liao, E.N. Eaton, A. Ayyanan, A.Y. Zhou, M. Brooks, F. Reinhard, C.C. Zhang, M. Shipitsin, L.L. Campbell, K. Polyak, C. Brisken, J. Yang, R.A. Weinberg. The epithelial-mesenchymal transition generates cells with properties of stem cells. Cell, 133 (2008), 704-715.

[23] F. Michor. Mathematical models of cancer stem cells. J. Clin. Oncol., 26 (2008), 2854-2861.

[24] C.A. O’Brien, A. Pollett, S. Gallinger, J.E. Dick. A human colon cancer cell capable of initiating tumour growth in immunodeficient mice. Nature, 445 (2007), 106-110.

[25] M.Z. Ratajczak, B. Machalinski, W. Wojakowski, J. Ratajczak, M. Kucia. A hypothesisfor an embryonic origin of pluripotent Oct-4(+) stem cells in adult bone marrow and other tissues. Leukemia, 21 (2007), 860-867.

[26] T. Reya, S.J. Morrison, M.F. Clarke, I.L. Weissman. Stem cells, cancer, and cancer stem cells. Nature, 414 (2001), 105-111.

[27] L. Ricci-Vitiani, D.G. Lombardi, E. Pilozzi, M. Biffoni, M. Todaro, C. Peschle, R. De Maria. Identification and expansion of human colon-cancer-initiating cells. Nature, 445 (2007), 111-115.

[28] I. Roeder, M. Herberg, M. Horn. An ”Age” structured model of hemapoietic stem cell organization with application to chronic myeloid leukemia. Bull. Math. Biol., 71 (2009), 602-626.

[29] S.K. Singh, I.D. Clarke, M. Terasaki, V.E. Bonn, C. Hawkins, J. Squire, P.B. Dirks. Identification of a cancer stem cell in human brain tumors. Cancer Res. 63 (2003), 5821-5828.

[30] H. Smith. An introduction to delay differential equations with applications to the life sciences. Springer, New York, 2010.

[31] F. M. Watt, B. L. Hogan. Out of Eden: stem cells and their niches. Science, 287 (2000), $1427-1430$

[32] R.A. Weinberg. The biology of cancer. Garland Science, New York, 2007. 
[33] A. D. Whetton, G. J. Graham. Homing and mobilization in the stem cell niche. Trends Cell Biol., 9 (1999), 233-238

[34] L. Wolpert. Positional information and the spatial pattern of cellular differentiation. J. Theor. Biol., 25 (1969), 1-47

[35] W.A. Woodward, M.S. Chen, F. Behbod, M.P. Alfaro, T.A. Buchholz, J.M. Rosen. WNT/beta-catenin mediates radiation resistance of mouse mammary progenitor cells. Proc. Natl. Acad. Sci. USA 104 (2007), 618-623.

[36] V.P. Zhdanov. Effect of cell-cell communication on the kinetics of proliferation and differentiation of stem cells. Chemical Physics Letters, 437 (2007), 253-256.

[37] S. Zhang, C. Balch, M.W. Chan, H.C. Lai, D. Matei, J.M. Schilder, P.S. Yan, T.H. Huang, K.P. Nephew. Identification and characterization of ovarian cancer-initiating cells from primary human tumors. Cancer Res., 68 (2008), 4311-4320. 